\title{
A new method of multi-beam real-time attitude compensation data processing
}

\author{
Zhang Yonghou ${ }^{1}$, Xiao Fumin ${ }^{*}$, Jin Shaohua ${ }^{1}$, and Bian Gang ${ }^{1}$ \\ ${ }^{1}$ Department of Military Oceanography and Hydrography and Cartography, Dalian Naval Academy, 116018 Dalian, China
}

\begin{abstract}
The traditional method for processing multi-beam real-time attitude compensation data did not consider the influence of attitude compensation error, which left attitude residual in data. So a new method for processing multi-beam data of real-time attitude compensation was proposed. By studying the nature of attitude compensation error, the calculation method of systematic attitude compensation error was put forward. The concept of correction threshold was introduced and the specific determination method was given. Finally, combining with the principle of real-time attitude compensation, a systematic attitude compensation error correction method, which makes use of a tracking algorithm considering attitude to make secondary attitude correction, was proposed, and the specific processing flow was given. By comparing the data processing accuracy of the tradition method and the new method, the results showed that the new method can effectively reduce the influence of systematic attitude compensation error, and significantly improve the data processing accuracy.
\end{abstract}

\section{Introduction}

Attitude correction is one of the most important links in multi-beam data processing. Its main purpose is to eliminate or weaken the impact of instantaneous attitude changes during the measurement process on obtaining high-precision position information and depth information at the sounding point. At present, there are mainly two methods for attitude correction in multi-beam measurement: the first is to use attitude sensors to collect attitude data synchronously during the multi-beam measurement process, and attitude correction is performed in the data post-processing stage; the second is to use the attitude data previously obtained by the sensor to estimate the attitude information of the next ping according to a specific algorithm during the multi-beam measurement process and use this information to perform real-time attitude compensation, that is, real-time attitude correction during the measurement process. And in the post-processing, attitude correction is no longer performed ${ }^{[1-2]}$, otherwise it will cause repeated correction errors.

Since the first method does not perform attitude compensation during measurement, the array is continuously distorted and deflected with the instantaneous attitude change, resulting in the phenomenon of submarine measurement strip bending and uneven distribution of measurement points (as shown in Figures 1a and 1c). It is difficult to control the overlap rate between adjacent strips and affect the measurement efficiency. Because of the real-time attitude compensation, the second method has more regular changes in the seabed cover strips, and the water depth points are evenly distributed ${ }^{[3-4]}$ (as shown in Figures $1 \mathrm{~b}$ and 1d). However, due to the limitations and diversity of the attitude estimation methods of different multi-beam, there may be an error between the predicted value and the true attitude value, and this error will affect the accuracy of the sounding point location.

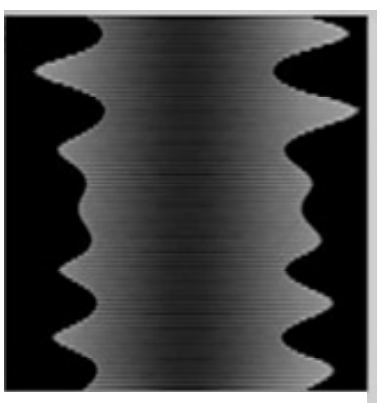

(a) without roll compensatio

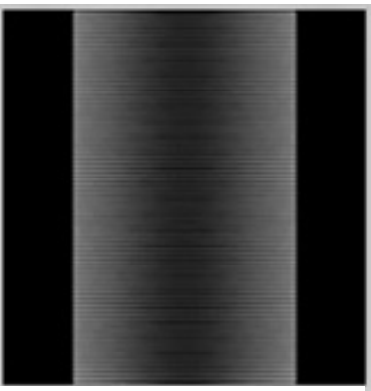

(b) with roll compensation

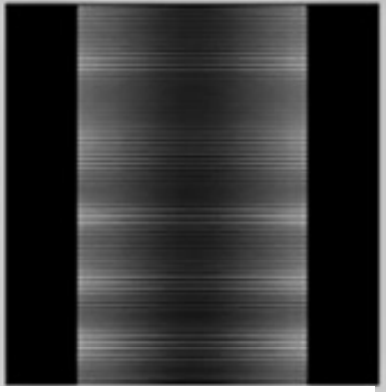

(c) without pitch compensation

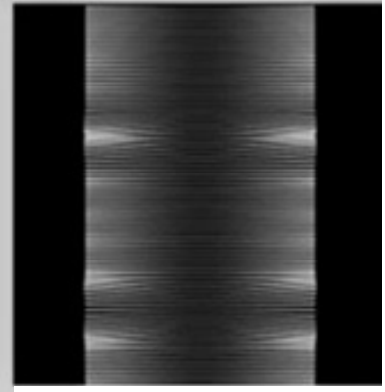

(d) with pitch compensation

Fig. 1. Distribution of seabed strips before and after compensation

Corresponding author: XIAO Fumin, PhD, associate professor. E-mail: xiaofum@163.com 
Yunta[3] briefly described the principle of real-time attitude compensation, demonstrated its data characteristics through simulation data, and further elaborated the advantages and disadvantages of the model; Ruoting[5] proposed a multi-beam sounding method that uses measured attitude data for roll stabilization, and the experimental results are ideal; Lingfeng[6] proposed a multi-beam sounding motion compensation method using combined inertial navigation system data, which achieved the desired accuracy; Based on Kiesel's[7] research, Fucheng[8] used phase shift beam-forming technology to control beam pointing and frequency difference to control the phase shift of each array element, and designed a new motion compensation technology for multi-beam sounding system.

Motion compensation has been widely used in various fields of marine surveying and mapping[9-12] , however, the current research is mainly aimed at the improvement of the real-time compensation working mode, and the processing method of compensation error data is rarely discussed further. Although post-measure attitude processing can no longer be performed in real-time attitude compensation mode, for data that may have compensation errors, reasonable secondary attitude correction is also a way to improve the accuracy of the measured data. Therefore, it is necessary to research on the multi-beam real-time attitude compensation data processing method based on the traditional real-time attitude compensation operation mode.

\section{Analysis of traditional real-time attitude compensation data processing problems}

Multi-beam real-time attitude compensation measurement is a new working mode introduced by the new generation of multi-beam. Its core is to transfer the traditional post-measurement attitude correction to the time of measurement, and no attitude processing is performed after the measurement. During the navigation of the measuring vessel, due to the influence of waves and swells on the hull, the transmitting attitude and the receiving attitude of the transducer change in real time. At this time, the ideal measurement state of the multi-beam is destroyed, and the spatial homing of the beam will produce a misalignment. Real-time attitude compensation can well eliminate the phenomenon of band bending and uneven distribution of measurement points caused by bad sea conditions. It is mainly based on attitude measurement values to estimate the attitude of the future moment through a specific algorithm. The reverse compensation based on the estimated attitude data can keep the multi-beam always in the ideal working state, and finally realizes the control of the beam distribution. However, the algorithms used by different instruments are different, so the estimation accuracy is also high or low. One thing to be reminded is that the manufacturers of this estimation method rarely have detailed descriptions.

Current mainstream multi-beam systems all have roll and pitch compensation functions, and a few of them also have heading compensation functions. Since heading errors have little effect on water depth, this paper mainly studies the effects of roll and pitch compensation errors. Here, the attitude compensation error is defined as the difference between the estimated value of the attitude and the true value. Because the attitude observation is accidental, and the estimation method also has systematic deviation, it can be considered that the attitude compensation error is accidental and systematic at the same time.

$$
\left\{\begin{array}{c}
\Delta(r, p)_{c}=\left|(r, p)_{t}-(r, p)_{c}\right| \\
\Delta(r, p)_{c s}=\left|\Delta(r, p)_{m}-\Delta(r, p)_{c}\right|
\end{array}\right.
$$

In the formula, $(r, p)_{t},(r, p)_{c}$ and $(r, p)_{m}$ are the true value, compensation value, and measured value of roll and pitch, respectively; $\Delta(r, p)_{c}$ is the attitude compensation error; $\Delta(r, p)_{c s}$ is the systematic part of the attitude compensation error.

In the traditional real-time attitude compensation data processing method, attitude correction is not performed after measurement. Therefore, the attitude compensation error introduced due to the defect of the estimation method is the main reason for the reduction of data accuracy in this processing mode. Due to the real-time nature of the multi-beam measurement, it cannot repeat redundant observations of the attitude, and thus cannot eliminate the accidental error in the attitude data by the adjustment method. Therefore, the following will mainly study how to use data processing to weaken the impact of the systematic part of the attitude compensation error on the data quality.

\section{New method of real-time attitude compensation data processing}

The main advantages of real-time attitude compensation are point distribution rules and high measurement efficiency. If two attitude corrections are applied to all data at the same time, it will inevitably reduce the data processing efficiency. And within the allowable range of error, it does not make much sense to correct the data with smaller compensation error. Therefore, in order to improve the accuracy of data processing while ensuring processing efficiency, the correction threshold is introduced as the basis for judging whether to make secondary corrections, that is, only secondary attitude correction is performed on data whose systematic attitude compensation error exceeds the threshold.

\subsection{Determination of correction threshold}

Using the definition of uncertainty in different water depth conditions in the IHO S-44 specification to constrain the coordinate error of the sounding point[13]. Define an effective beam as a beam that meets the requirements of both the horizontal and vertical 
uncertainty limits. The effective beam ratio is the ratio of the number of beams satisfying the requirement to the total number of beams. The multi-beam technical measurement specification published by China in 2003 requires that the line spacing be at least $20 \%$ overlapping between adjacent lines [14]. Therefore, it can be considered that at least $80 \%$ of the effective beams must be guaranteed during the measurement to meet the measurement requirements.

First, according to the actual situation, combined with the error propagation law, determine the proportion of the horizontal error and vertical error caused by the attitude compensation error in the total error(defined as horizontal ratio and vertical ratio). Secondly, assuming that the water depth is constant and known, the system has a known difference in roll and pitch compensation. According to the number of beams and the beam allocation angle of the multi-beam system, combined with the acoustic profile data, the horizontal positioning error and vertical distance error of different beams in a ping are calculated. The uncertainty limit is used to determine whether it is an effective beam, and the effective beam ratio at this time is further determined. At this water depth, the effective beam ratio can be obtained by substituting different roll and pitch compensation differences. Compared with rolling, pitching has the characteristics of small change interval and change law. In addition, the pitch error has little effect on the measurement results, so the pitch compensation difference can be set as a fixed value, calculating with different roll compensation differences, and the roll compensation difference can be used as the judgment threshold. Finally, the roll compensation difference with an effective beam ratio of $80 \%$ is regarded as the correction threshold at this water depth, and the threshold at different depths can be calculated by this method. Here, calculating the threshold value of the approximate water depth of the measurement area is enough.

\subsection{Correction of systematic attitude compensation errors}

After determining the correspondence between the depth in the survey area and the correction threshold, the attitude observation value and compensation value of each ping can be extracted, and calculate their systematic attitude compensation errors, that is the roll compensation difference $\Delta r_{c s}$ and the pitch compensation difference $\Delta p_{c s}$. The central beam water depth is used to determine the roll compensation error correction threshold. If the threshold is exceeded, a second attitude correction is performed on it, otherwise the correction is no longer performed. For the over-limit ping, regarding the compensation difference $\Delta r_{c s}$ and $\Delta p_{c s}$ as observation of the roll and pitch, the second attitude correction is performed on the basis of the original measurement data.

Real-time attitude compensation is essentially to correct the beam direction, so that the beam footprint always covers the ideal area, so as to achieve the control of the sounding point position [3]. Based on the same idea, the second attitude correction also directly affects the attitude on the beam incidence angle, that is, the accurate sound tracking algorithm that takes into account attitude is used for correction.

To deal with the attitude compensation error, the principle of first rotation and then tracking should be adopted. However, the observation value of the attitude sensor cannot be directly used in the construction of the rotation matrix. According to related researches [15]:

$$
\left.\begin{array}{c}
\alpha=\sin ^{-1}(\sin r / \cos p) \\
\beta=P
\end{array}\right\}
$$

According to equation (2), the rotation angle $\beta$ of the array around the $\mathrm{Y}$ axis is equal to the pitch $p$, but the rotation angle $\alpha$ of the array around the $\mathrm{X}$ axis is not equal to the roll $r$, and the size of angle $\alpha$ is determined by both roll and pitch. Therefore, attitude observation values cannot be directly used for correction, and the coupling effect between attitudes must be considered.

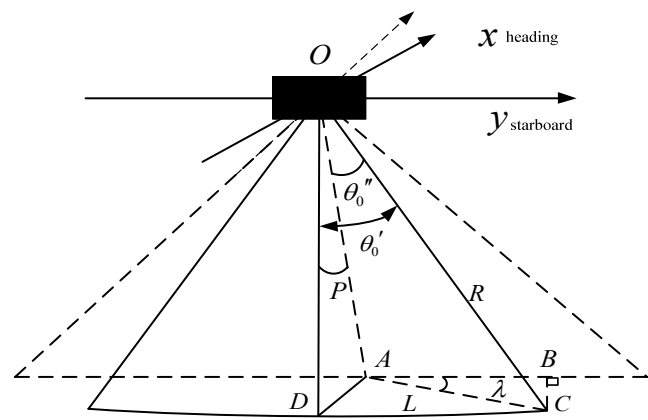

Fig. 2. Derivation of beam incident angle

As shown in Figure 2, $O A$ is the vertical axis, $p$ is the pitch, $R$ is the slant distance, the dashed line is the sound ray propagation surface under ideal conditions, and the solid line is the actual sound ray propagation surface affected by the attitude. The relations in figure 2 can be described by equation (3) as follows:

$$
\theta_{0}^{\prime}=\theta_{0}+\alpha=\theta_{0}+\sin ^{-1}(\sin r / \cos p)
$$

At the same time, the another equation as follows:

$$
O A=O C \cos \theta_{0}^{\prime} \cos p=R \cos \theta_{0}^{\prime \prime}
$$

Therefore, considering the combined effects of roll and pitch, the actual beam incidence angle $\theta_{0}^{\prime \prime}$ is as follows:

$$
\begin{aligned}
\theta_{0}^{\prime \prime}= & \cos ^{-1}\left(\cos \theta_{0}^{\prime} \cos p\right)= \\
& \cos ^{-1}\left(\cos \left(\theta_{0}+\sin ^{-1}(\sin r / \cos p)\right) \cos p\right)
\end{aligned}
$$

According to the refraction theorem, it can be found that under the influence of pitch, the sound ray propagation surface changes from a flat surface to a curved surface [3]. And the deflection of the array along 
the track direction produces a horizontal angle $\lambda$, which is $\angle C A B$ in the figure2. It can be described by equation (6) as follows:

$$
\lambda=\cos ^{-1}(A B / A C)=\cos ^{-1}\left(\sin \theta_{0}^{\prime} / \sin \theta_{0}^{\prime \prime}\right)
$$

Assuming that the transducer installation is error-free, the actual incidence angle is used to track the constant gradient sound rays to obtain the vertical depth $Z$ and the lateral offset distance $L$, and the beam spot coordinates in the vessel frame system (VFS) can be obtained with the horizontal angle $\lambda$ :

$$
\left[\begin{array}{c}
X \\
Y \\
Z
\end{array}\right]_{V F S}=\left[\begin{array}{c}
X_{0}+L \sin \lambda \\
Y_{0}+L \cos \lambda \\
Z_{0}+Z
\end{array}\right]
$$

In the formula, $\left(X_{0}, Y_{0}, Z_{0}\right)$ is the coordinate of the origin of the transducer coordinate system under the vessel frame system .

The multi-beam real-time attitude compensation data processing steps are as follows:

(1) Determine the horizontal and vertical proportions according to the actual measurement situation, and combine the multi-beam measurement parameters, acoustic profile data, water depth range of the measurement area and other data to calculate the correction threshold at different depths.

(2) Extract the measurement data collected by the real-time attitude compensation operation, calculate the systemic attitude compensation error per ping, and obtain the corresponding roll compensation difference and pitch compensation difference.

(3) Determine the correction threshold using the central beam water depth of each ping, and decide whether the roll compensation difference exceeds the limit.

(4) If it exceeds the limit, the difference between the horizontal and vertical compensation of the over-ping ping is regarded as the observation value of the horizontal and vertical attitude, and the secondary attitude correction is performed. If the limit is not exceeded, no correction will be performed.

(5) Perform steps (2)-(4) for each ping data until the end.

\section{Simulation test and analysis}

\subsection{Threshold calculation}

The study found that the same attitude compensation error has a greater impact on the vertical measurement results, but has less impact on the horizontal position and is generally much smaller than the limit difference requirements. Therefore, the vertical error can be used as the main judgment basis. Assuming that the system beam opening angle is $128^{\circ}$, the number of beams is 256 , and the water depth is set to 40 meters, 100 meters, and 300 meters, respectively (see Figures $2 \mathrm{a}, \mathrm{b}$, and c), the roll compensation differences are $0.02^{\circ}, 0.04^{\circ}, 0.06^{\circ}$ 、 $0.08^{\circ}$ and $0.1^{\circ}$ (marked with different symbols in Figure 2). The pitch compensation difference is $0.1^{\circ}$, the roll and pitch measurement errors are both $0.01^{\circ}$, the proportion of horizontal and vertical is $10 \%$. Combine the acoustic profile data to calculate the beam vertical error at different incident angles. The results are shown in Figure 2. The abscissa is the beam incidence angle, the ordinate is the vertical error, and the dashed line is the $10 \%$ vertical uncertainty limit at the corresponding water depth.

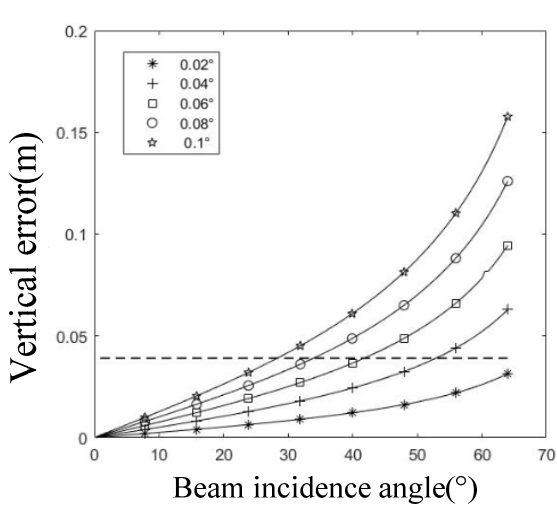

(a)

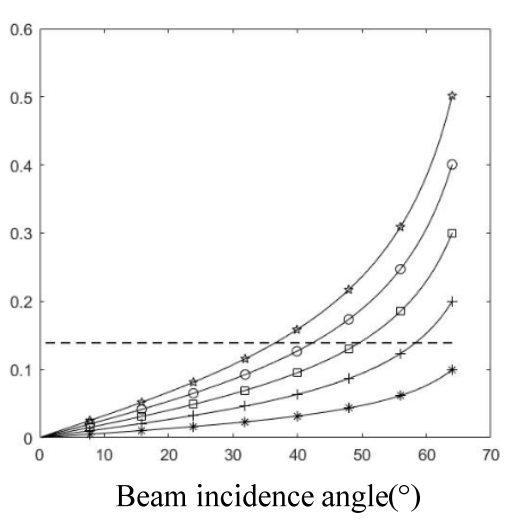

(b)

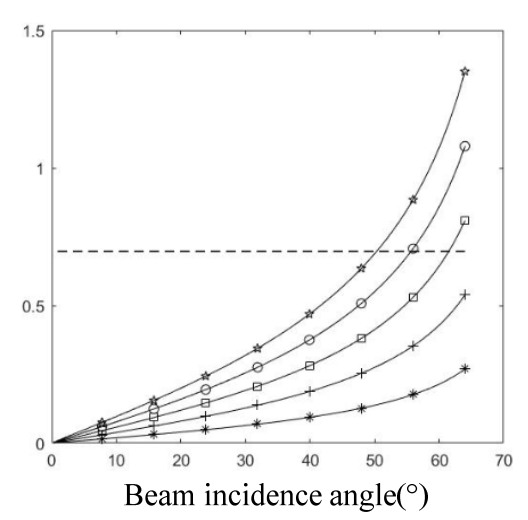

(c)

Fig. 3. Vertical error of $40 \mathrm{~m}, 100 \mathrm{~m}$ and $300 \mathrm{~m}$

In Figure $3 \mathrm{a}$, the effective beam ratios at a water depth of 40 meters are: $100 \%, 82.0 \%, 64.8 \%, 53.1 \%$, and $44.5 \%$. Set the value range of the roll compensation difference to $0.04^{\circ} \sim 0.06^{\circ}$, and use the approximation principle to gradually obtain the roll compensation difference with an effective beam ratio of $80 \%$, which is $0.045^{\circ}$. At the same time, the difference in roll compensation of $80 \%$ of the effective beams at $100 \mathrm{~m}$ and $300 \mathrm{~m}$ water depth can be obtained as $0.065^{\circ}$ and 
$0.112^{\circ}$. Using the above method, calculate the correction threshold for the roll compensation difference of $80 \%$ of the effective beam at a water depth of $5-300$ meters. The results are shown in Figure 4. The abscissa is the water depth, and the ordinate is the correction threshold of roll compensation differences.

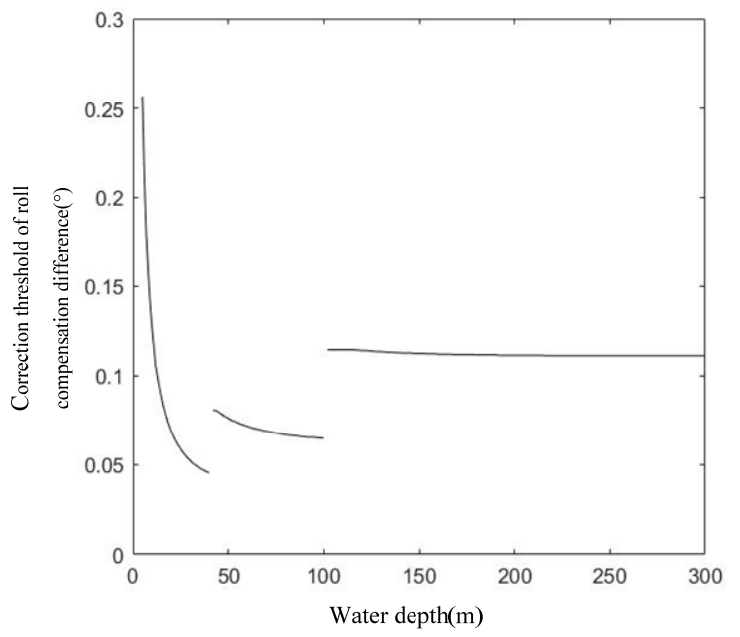

Fig. 4. Correction threshold of roll compensation difference

The vertical uncertainty in the S-44 specification has different accuracy requirements for different water depth, so the correction threshold also shows a variety of different forms. As can be seen in Figure 4, the shallower the water depth, the greater the threshold change rate, while the threshold is almost unchanged when the water depth is greater than 100 meters. Which show the sensitivity of the correction threshold to changes in water depth gradually weakens as the water depth increases. In this paper, the $10 \%$ uncertainty limit is used as a constraint, and the ratio can be adjusted according to the actual situation of measurement. At the same time, the threshold correction will be affected by the acoustic profile data. The threshold values of different systems will be various, but the overall change law is basically the same.

\subsection{Simulation test}

In order to verify the effectiveness of the method in this paper in improving the accuracy of data processing, the following simulation experiments were carried out: the system beam opening angle was $128^{\circ}$, the number of beams was 256, and the roll and pitch measurement errors were both $0.01^{\circ}$. Assuming that the difference of pitch compensation per ping in the system is $0.1^{\circ}$, and the difference of roll compensation is the correction threshold corresponding to the depth of the central beam, that is, the attitude compensation error per ping just exceeds the limit. Set the water depth to 5-300 meters, the depth and the correction threshold meet the corresponding relationship in Figure 4, combined with acoustic profile data to calculate the measurement data of real-time attitude compensation operation mode at different depths. The traditional real-time attitude compensation data processing method and the method in this paper are used to process the simulation data separately, so the effectiveness of the processing method can be judged by comparing the accuracy of the data processing. The processing results are shown in Figure 5. Here, the average vertical error is defined as the average value of the vertical errors of all beams in one ping, and the average horizontal error is the same.

In Figure 5a, the solid line and the dashed line respectively represent the average value of the vertical error of the data processed by the traditional method and the new method. It can be seen that the vertical error of the data processed by new method is smaller, which is improved to a greater extent than the traditional method, and the greater the depth, the more obvious the effect of improving vertical accuracy. At a water depth of 50 meters, the new method can reduce the vertical error by about $60 \%$ (the vertical error caused by the compensation error), and the value reaches about $73 \%$ at 200 meters. Figure $5 \mathrm{~b}$ is a comparison of the average horizontal error of the two methods. The new method also effectively reduces the horizontal positioning error. At a depth of 50 meters, it can reduce the horizontal error by about $48 \%$ (the horizontal positioning error caused by the compensation error). At 200 meters, it can be reduced by about $64 \%$. 


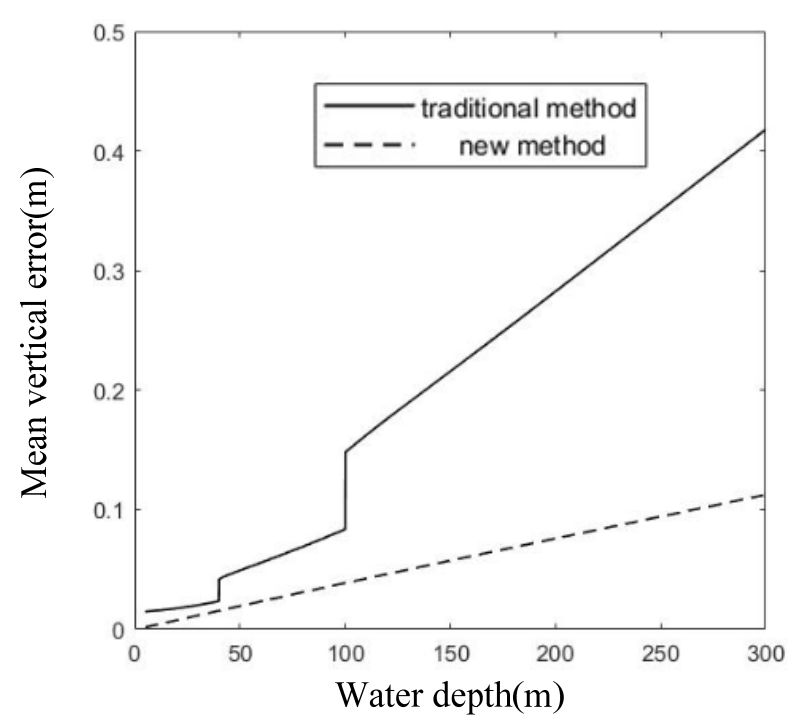

(a)

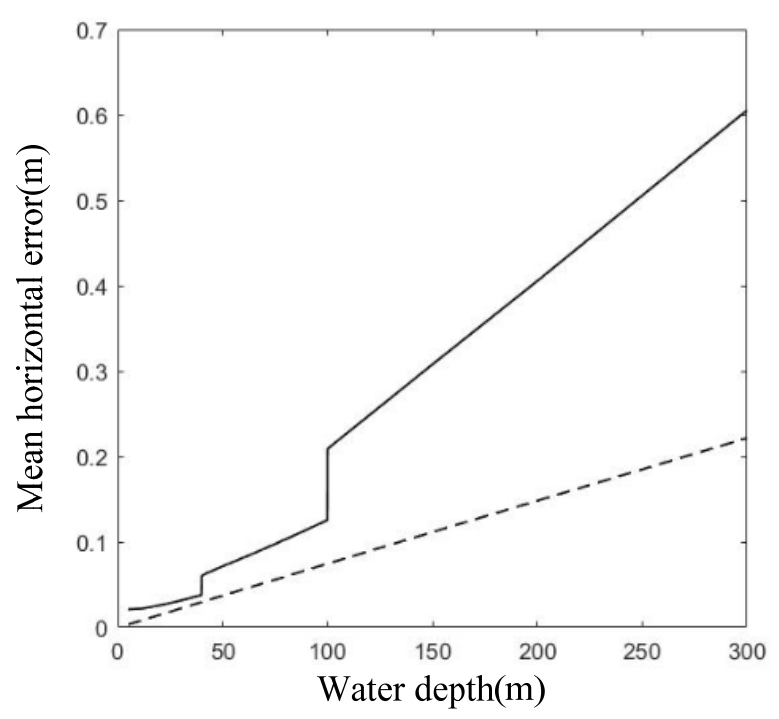

(b)

Fig. 5. Comparison of data processing accuracy between traditional method and new method

In the above test, the correction threshold at the central beam depth was used as the roll compensation difference, and using a fixed pitch compensation difference for simulation calculation. However, in practical work, due to the limitations of the estimation method and the complexity of the actual situation, it is generally difficult to control the attitude compensation error very well, so it is easy to produce a large systematic attitude compensation error. Therefore, in the actual situation, the method in this paper can obtain a better improvement effect than the above simulation results, that is, the improvement effect on the data accuracy of the method in this paper increases with the increase of the attitude compensation error.

\section{Conclusion}

This paper proposed a new multi-beam real-time attitude compensation data processing method. First, based on the measurement data and related measurement parameters, calculate the correction threshold within the water depth of the measurement area. Then sequentially determine whether the data exceeds the limit, for the over-limit data, attitude correction processing is performed by using a sound-tracking algorithm that takes into account attitude, while there is no correction for normal data. Through simulation experiments, the correction threshold at the central beam depth is used as the roll compensation difference for sounding data simulation, then the traditional method and the new method are used to process the simulation data, and the data processing accuracy of the two methods is compared. The experimental results show that the method in this paper significantly improves the data processing accuracy compared with the traditional method, and the improvement effect on the data accuracy gradually increases with the increase of the attitude compensation error, which has important reference value and practical value for the refined processing of multi-beam data.

\section{Acknowledgments}

Foundation Support: The National Natural Science Foundation of China $(41876103,41576105)$

\section{References}

1. L. Jiabiao, W. Xiaobo, H. Zugen, et al. Multi-beam sounding surveying: principle, technology and data processing methods[M]. Beijing: Ocean Press, 1999.

2. Z. Jianhu, L. Jingnan. Multi-beam bathymetric surveying and image processing[M]. Wuhan: Wuhan University Press, 2008.

3. T. Yunta. Sector-specific Beam Pattern Compensation for Multi-sector and Multi-swath Multi-beam Sonars[D]. The University of New Brunswick,2011.

4. W. Ziyin, Y. Fanlin, L. Xiaowen, et al. High Resolution Submarine Geomorphology[M]. Beijing: Science Press, 2017.

5. C. Ruoting, L. Xiaodong, L. Zhiyu, et al. A bathymetric algorithm based on roll compensation for multi-beam echo sounder[J]. Technical Acoustics, 2013,32(05):368-372.

6. Z. Lingfeng. The motion compensation of multi-beam bathymetric system based on integrated navigation system[C]. China Institute of Inertial Technology. Proceedings of the conference on dynamic development direction of inertial technology. China Institute of Inertial Technology. China Institute of Inertial Technology,2012:51-55.

7. K C. Kiesel. A New Pitch/Yaw Stabilized Bathymetric Survey System[J]. IEEE,2000,201-202.

8. B. Fucheng. The study on movement compensation new technique and hardware design of multi-beam swath bathymetry[D]. Harbin Engineering 
University,2007.

9. Z. Suoping, Z. Chuntian. Motion compensation for wave measurements using up-looking sonar form underwater moving platform[J].Transducer and Microsystem Technologies, 2009,28(04):59-62.

10. Y. Haiting, L. Jiyuan, Z. Chunhua. Motion Compensation of Synthetic Aperture Sonar Based on Inertial Measuring System[J]. Journal of Electronics \& Information Technology, 2007(01):63-66.

11. Z. Huwanling, D. Hongbing. Study on Error Compensation for U- turn Attitude Angle Measurement Based on MEMS Inertial Technology[J]. Guizhou Science, 2012,30(01):70-72.
12. T. Weifeng, Y. Bingcheng, L. Kai. Research on Identification and Compensation of Attitude Measurement Errors for SINS[J]. Chinese Journal of Inertial Technology, 1997(02):14-19.

13. S-44 5th Edition. IHO Standards for Hydrographic Surveys[S]. Monaco: International Hydrographic Organization,2008.

14. Technical Requirements for Multi-beam Bathymetry [S].Naval Staff Navigation Assurance Bureau,2003.

15. H. Linbang, Z. Jianhu, Z. Hongmei, et al. A precise multi-beam sound ray tracking method taking into account the attitude angle[J]. Journal of Harbin Engineering University,2015,36(01):46-50. 